\title{
How to Use the Method of Multivariate Statistical Analysis Into the Equipment State Monitoring
}

\author{
Chunhua Yang \\ Baoshan University, Baoshan, 678000, China
}

Keywords: Multivariate statistical analysis, Equipment state, ICA, KPCA, Automobile gearbox

\begin{abstract}
The method of multivariate statistical analysis is a branch of classical statistics, is a comprehensive analysis method, it can also have each other for multiple objects and the index of correlation analysis, parsing the statistical rule between them, at present has been widely applied to agricultural scientific research and industry. Using multivariate statistical analysis can be for condition detection and accurate diagnosis of industrial equipment, find their potential failure. In this paper, it based on independent component analysis ICA method of multivariate statistical analysis on the automobile gear box of equipment state monitoring and analysis.
\end{abstract}

\section{Introduction}

Through the different parts of the equipment acquisition signal can for multi-channel monitoring of running status of equipment, in order to get full state information. In order to further conducive to online monitoring for the equipment efficiency, promote multipoint, state of multi-sensor acquisition ability, should choose the application of multivariate statistical analysis methods. It can be real-time monitoring of equipment for multidimensional single analysis of measurement data processing, and unique statistical information from multidimensional signals, more clear expression of the existing equipment real-time characteristics and possible failure state.

\section{Independent component analysis equipment transient detection method analysis of ICA}

Independent component analysis ICA can surround equipment vibration source analysis equipment real-time state, so it has high application value in the field of vibration analysis. In order to more conducive to running equipment vibration analysis research, many scholars have spun off from the equipment vibration, vibration components separately it covered the vibration period of composition, smooth transient components and cycle components and so on. So the ICA and blind source separation BSS are different, it from another Angle to extract the vibration source of statistical information and vibration analysis of research and application. For example in the process of the phase spectrum, which reflects the natural vibration signals in transient testing under the capture of the vibration of the equipment image edge, impact vibration signal and sound signal instantaneous pulse showed great ups and downs, they cater to the basic characteristics of ICA in statistics, when the device runs in a different state and produces different vibrational state and statistical structure, equipment transient detection based on ICA will reflect the value of it [1].

\section{The ICA transient detection method}

On measurement equipment, measuring signal generated by the transient components will keep on time, according to the band width and so on index exists in the testing environment, which is very valuable for ICA instantaneous detection of feature information. Automobile gearbox vibration signals, for example, it is embodied in the transient components of general gear there have been some damage, deformation or fracture of partial failure, so this means the transient components of automobile gearbox vibration analysis is conducive to maintaining the stability of its operation. But considering the transient vibration signal analysis will have serious noise, it obscures the gear due to vibration of information, so this paper puts forward a low SNR environment testing equipment vibration signal transient detection method. The method mainly includes three steps, will initially be one dimensional signal segmentation and used for training of independent component analysis ICA. Then apply maximum a posteriori probability assessment of vibration signal of shrinkage function is 
derived, and with the help of ICs coefficient, equipment vibration signal de-noising. Finally rebuild the transient signal through contraction coefficient completely basis function.

\section{The basic principle of detection}

For automobile gear box running state monitoring, transient detection is of great significance; it can be comprehensive analysis of characteristics of the vibration of the gear box, extract the vibration signal analysis and find the possible partial failure. In general, the gear box of partial failure often characterized by regular periodic pulse, it's just cater to the ICA transient signal in the super Gaussian statistical features of the composition analysis. So as long as after ICA transform, segmentation and collates the vibration signal, vibration signal can be a new independent non-Gaussian coefficient linear combination. , on the other hand, can also through the use of peculiar to the ICs coefficient sparse feature to distinguish the gearbox noise existing in the signal and the transient signal, which is based on ICA in detecting transient signal transformation increases the statistical difference between the transient signal and noise signal, so they tell the transient component to be effective. In automotive gear box the running noise larger devices, extracting their noise should be first based on vibration signal with noise removing noise coefficient decomposition coefficient, then cut with noise by using ICA basis function decomposition coefficient of the reconstruction of transient components. Because vibration signals of high order statistics basis function can be cooperated by ICs coefficient sparse feature to sparse transient component of vibration signal, so the transient testing method is feasible, it can adapt to different situations of different noise signals, the adaptability is considerable. The application of transient detection method based on ICA car gearbox condition monitoring

After doing fatigue test for motor speed change gear box, use the ICA transient detection method for vibration analysis, which had proved that the method application effect in gear box running status monitoring. As shown in figure 1.

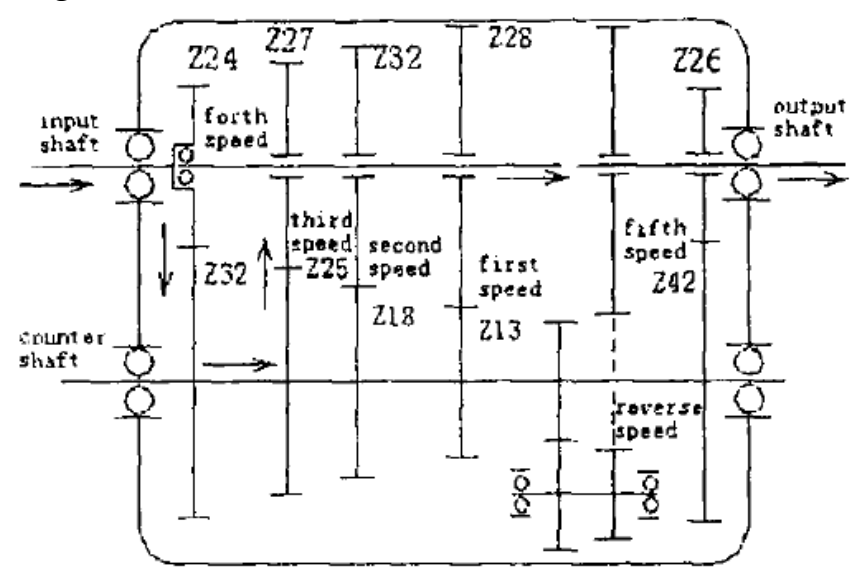

Fig. 1 The internal structure of car speed gear box

As shown in figure 1, the automobile transmission gear box there are five forward gears and one reverse gear. Vibration signals from the three gear box shell, and gained from gear accelerator sensor. Amplify the signal acquisition and storage to the computer system, the test sample frequency is $3500 \mathrm{~Hz}$. If the gear speed $2000 \mathrm{r} / \mathrm{min}$, load the load of $250 \mathrm{~N} / \mathrm{m}$, it is immediately to change gear according to automobile gear box to implement six levels of cyclic fatigue test. Experiment, the first three gear after basic normal state, but with minor wear and tear on the sixth gear, the serious wear and lead to change gear tooth broke, the number of gear engagement of $350 \sim 4$ million times. 

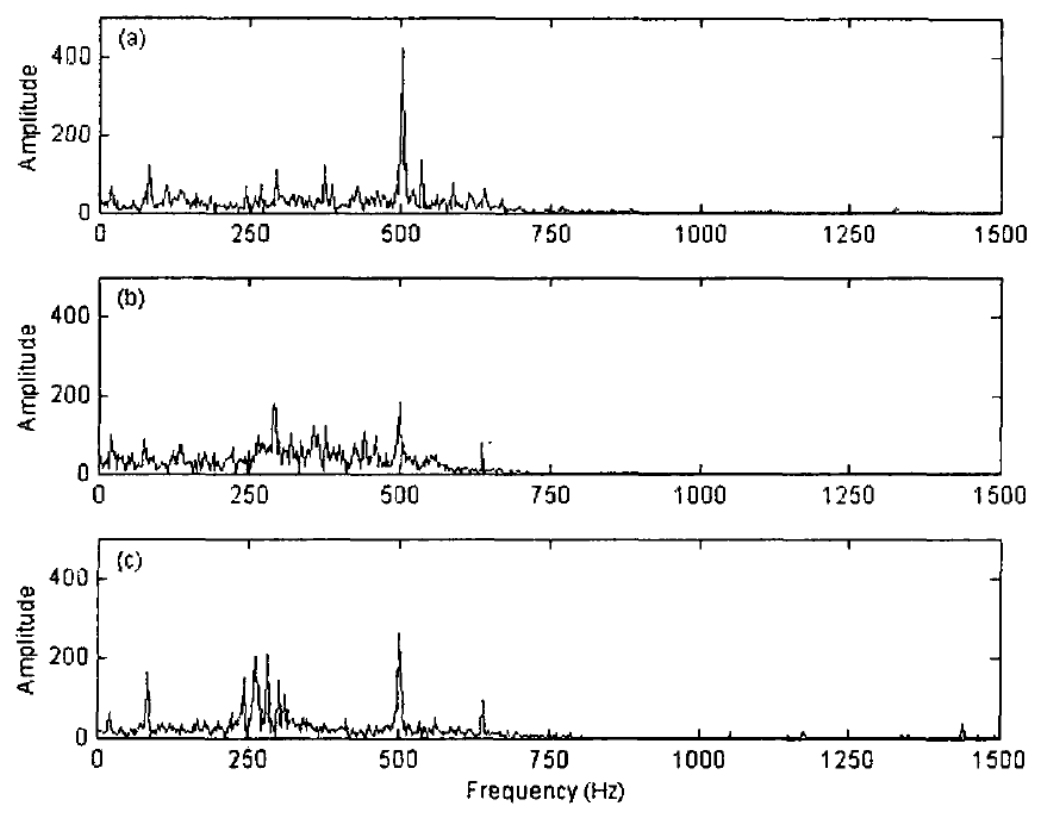

Fig. 2 Car speed gearbox vibration signal spectrum picture

As shown in figure 2, the above the graph is variable speed gear box in the normal operation of the 1 gear, the graph contains mild wear between $2 \sim 5$ gear, the bottom of the graph is serious wear of 6 gear failure modes. Due to noise pollution is serious, and definite exists in the process of gear vibration is analyzed on transient components, so first of all should be from the test confirmed the existence of the transient components in vibration signal, and then extract and effective application of it to the variable speed gearbox condition monitoring process.

Because there are three kinds of vibration signals, so first of all to define the signal separation size, rules of 15, as shown in figure 3.
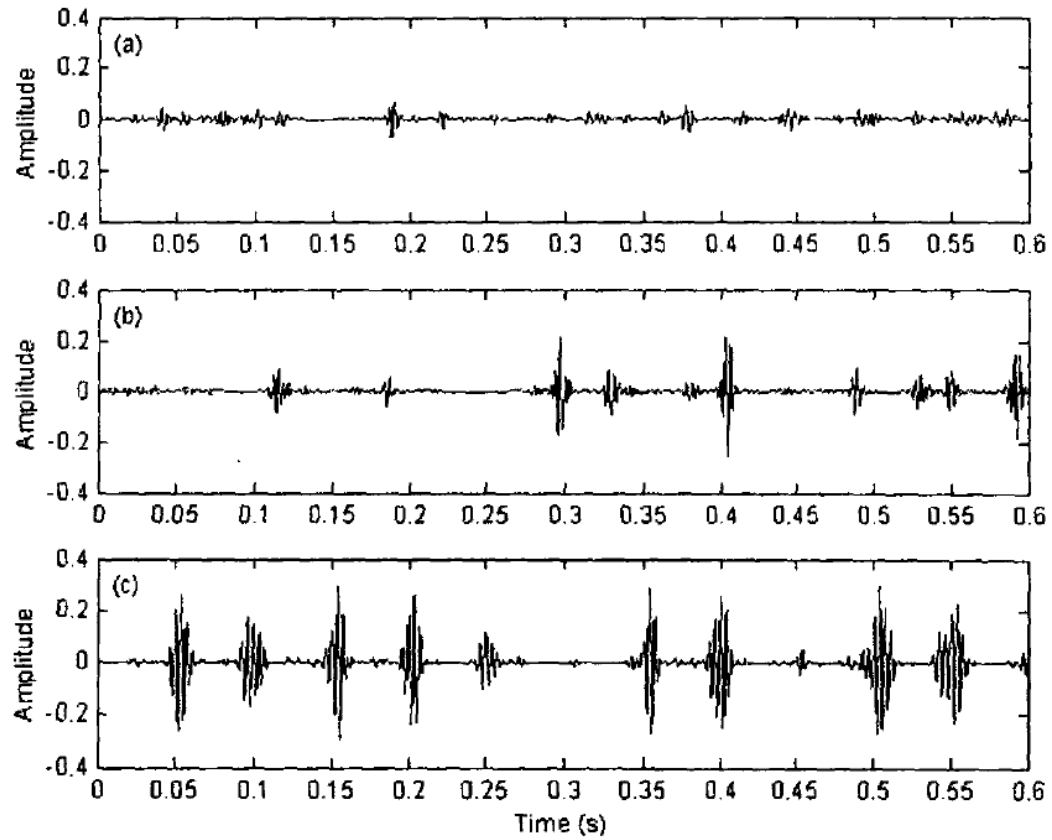

Fig. 3. Transient detection method based on ICA gearbox vibration signal under transient component diagram

In figure 3, 3 transient component image can clear change gear box under the three states of transient components, and the health status of change gear box in decline gradually. The improved transient signal amplitude, it means that the increasing transmission cycle regularity. As shown in figure 3 from top to bottom three picture, show the transient signal is weak irregular change of state. One of the most in the figure above the minimum amplitude, shows that the initial state of gear in the 
gearbox of wear is very good, basic no wears and tear. The second chart shows with the deepening of vibration, the cycle period of apparent after showed the amplitude increase and frequent phenomenon, gear has been mild wear at this time. See the bottom plan signal amplitude increases and regular, reflects obvious periodic transient components, it also suggests that the existence of transient pulse. At this time for serious wear stage, drive gear in the gearbox to broken teeth, the embodiment of partial failure is also evident in the transient components. So, 3 picture of the three kinds of transient signal under the amplitude change of periodic clear effective state of evolution, embodies the variable speed gearbox, and transient analysis can also be a good match to the test results, it proves that based on ICA transient detection method in mechanical equipment such as motor speed change gear box in the effectiveness and accuracy of application.

Moreover, it can also use the ICA transient detection methods to verify the equipment running status when the convergence of vibration signals. Because of serious wear and tear on the vibration signal after repeated transient testing, multiple test results found that overall transient signal amplitude is not obvious and irregular cycle performance, so it failed to clear reflect the characteristics of the change gear box of real-time state. So this also proves that the convergence of ICA method under gear serious wear and tear. It also tell us if the equipment running status using the ICA transient detection method, be sure to global detection is given priority to with the first test results, it can be the first time through the analysis of vibration signal good validation change gear box of the true state of [2].

\section{The feature extraction of equipment state monitoring in multivariate statistical}

Multivariate statistical characteristic analysis method can objectively reflect the sensitivity of the equipment in the running state and regularity, clustering classification diagnosis of equipment state. Tradition if according to the frequency domain and time domain wavelet domain to reflect the characteristics of the fault diagnosis generally need more number, but if use multivariate statistics method to extract, can not only reduce the characteristic number, also can improve the clustering classification diagnosis effect, it also suggests that multivariate statistical features can reflect inherent statistical data structure.

\section{Feature extraction}

There are more methods of feature extraction Multivariate statistical analysis, such as PCA and ICA, KPCA and so on. They can be extracted from multiple original special complete the characteristics of the new structure, after analyzing between the original truthfully reflect the diversity feature of statistical properties, like to independence, higher order statistics, nonlinear feature extraction is so and so on.

Any mechanical device will vibrate at run time, and it is complex and random noise signal, the basic cannot use time function. Would then use multivariate statistical feature extraction, the running status of equipment monitoring, real-time extraction can reflect the characteristics of the equipment state information signal. Using the variance of standardized feature extraction method can avoid the noise, the influence of such operating environment for more fair and objective signal analysis results, it is also a kind of pretreatment for the measured signal. After the pretreatment, measuring signal would be unit variance and zero mean standards. Equipment that be measured noise signal is converted to a set of one-dimensional discrete data (y1, y2, y n...), where $\mathrm{N}$ said sample length, then the data sample mean should be represented as:

$$
\bar{y}=\frac{1}{N} \sum_{i-1}^{N} y_{i}
$$

This sample variance should be as follows:

$$
S=\frac{1}{N} \sum_{i=1}^{N}\left(y_{1}-\bar{y}\right)^{2}
$$

So can get sample data using the variance of standardized analysis is: 


$$
y_{i}=\frac{y_{i}-\bar{y}}{\sqrt{S}}
$$

After the above a series of processes of pretreatment, found that the equipment are reflected in the time domain signal in different condition. And at the same time, through the analysis of the frequency domain of the signal under different condition, can also find some typical characteristics of band, they can reflect the device in different state, the development of the fault produced under different degree. So based on the time-frequency and frequency domain analysis, it can be extracted from the equipment vibration and noise characteristics of time domain and frequency domain index, using the maximum peak, root of the noise and vibration signal amplitude, RMS, absolute mean value, variance and the peak factor, waveform factor and so on domain and frequency domain for the eight relative spectral energy parameter to measure the signal characteristics are given for resolution, measuring the characteristic vector of signal should be expressed as $\left(F_{j} \mid j=1,2, \ldots, 16\right)$.

\section{The fault diagnosis based on multivariate statistical analysis method of internal combustion engine wear.}

Multivariate statistical analysis method can predict the factory internal combustion engine wear fault state, as far as possible to avoid economic loss for the factory, reduce the maintenance cost. Due to the internal combustion engine with oil inside channel mouth, so I can work through the channel's voice to reflect the internal combustion engine running state. In oil mouth set the microphone to monitor gas noise signal acquisition, this paper chose the existence of four kinds of fault clearance condition of noise signal to the analysis of internal combustion engine running state. Noise signal to select 80 groups, including 50 group used for training, 30 group was used to test the rest.

The state recognition of engine. Any extraction group of 16 in the noise signals, they represent the 16 time-frequency statistical characteristic index, they can be used to further characterization of the internal combustion engine connecting rod bearing model characteristics. According to the 80 training sample set to correspond to the corresponding statistical characteristic index.

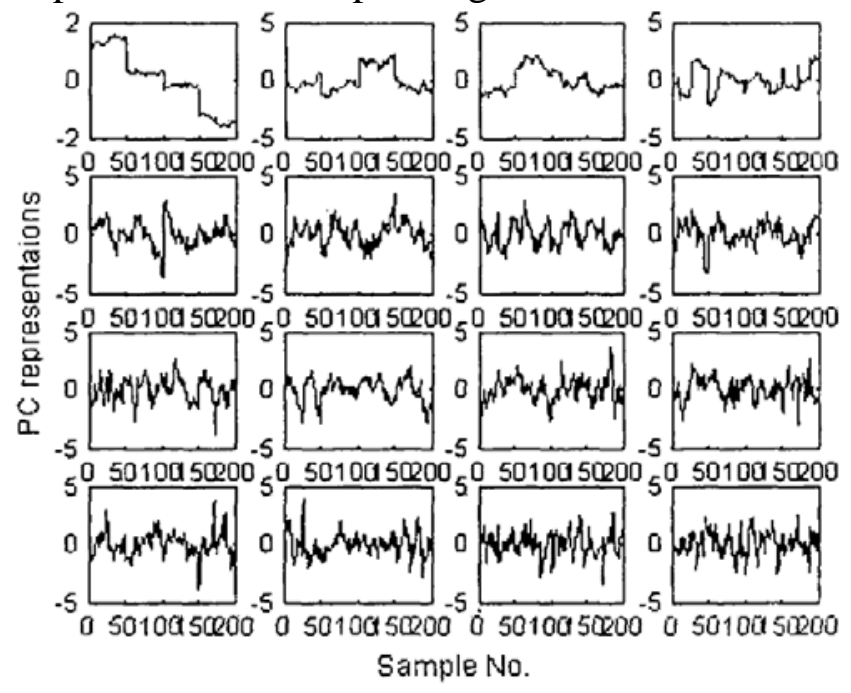

Fig. 4 Internal combustion engine groups of 16 PC features of noise signal representation

Seen in figure 4, 16 group said the characteristics of the noise signal to the performance of the internal combustion engine running state each are not identical, part reflects the regularity of stable state, the other part is the relative state is not sensitive, regularity is not obvious.

But using PCA method to extract the 16 training sample were analyzed, and the noise signal shown their principal component characteristics as shown in figure 4, you can see its principal component feature set each training sample of the original noise signal characteristics, these can show the internal combustion engine running state, and can be applied to internal combustion engine wear diagnosis. And the other parts of the principal component features can reflect the equipment itself under different condition of local wear change. So you can through the way of quantitative evaluation 
principal component characteristics to diagnose internal combustion engine wear diagnosis effect, namely the calculation one by one in 16 samples of the original noise characteristics and the related coefficient of the original PC characteristics, the calculation formula is: $\rho_{t h}^{16}=0.3519$. At this point to continue using the MCR detection method, on the PC characteristics of 16 samples of noise diagnosis, and linked with internal combustion engine wear diagnosis condition, can show that PC features and all the characteristics of noise signal with strong correlation, which can describe the equipment in 16 all state changes in the process of noise signal is represented by [3].

\section{Conclusion}

This article mainly based on multivariate statistical analysis methods such as ICA, KPCA and so on component analysis method to the complete state of industrial machinery and equipment monitoring and diagnosis, reflects the very good application effect. So PCA based on principal component analysis, independent component analysis ICA, kernel principal component analysis (KPCA and blind source separation BSS the four methods of multivariate statistical analysis of industrial equipment running condition monitoring application there are many, they are actively reflect the higher-order statistics under the equipment running status of the multidimensional measurement signal extraction and separation, have good development prospect in the industrial field, it is worth further study.

\section{References}

[1] Fu Deyin. The construction of multivariate statistical analysis method, inspection system. Journal of business and management,2009,(2):76-80.

[2] Qi Hhongquan. A comprehensive evaluation method of multivariate statistical analysis. University of Hunan,2010.

[3] He Qingbo. Multivariate statistical analysis in the diagnosis of equipment state monitoring research. University of Science and Technology of China.2007.75-90. 